\title{
Evaluation of comprehensive chromosome screening platforms for the detection of mosaic segmental aneuploidy
}

\author{
David Goodrich ${ }^{1}$ - Tongji Xing ${ }^{1} \cdot$ Xin Tao $^{2}$ • Agnieszka Lonczak ${ }^{2}$ - Yiping Zhan ${ }^{2}$ • \\ Jessica Landis ${ }^{2} \cdot$ Rebekah Zimmerman ${ }^{2} \cdot$ Richard T. Scott Jr ${ }^{1} \cdot$ Nathan R. Treff $^{1}$
}

Received: 5 January 2017 / Accepted: 11 April 2017 / Published online: 2 June 2017

(C) The Author(s) 2017. This article is an open access publication

\begin{abstract}
Purpose A subset of preimplantation embryos identified as euploid may in fact possess both whole and subchromosomal mosaicism, raising concerns regarding the predictive value of current comprehensive chromosome screening (CCS) methods utilizing a single biopsy. Current CCS methods may be capable of detecting subchromosomal mosaicism in a trophectoderm biopsy by examining intermediate levels of segmental aneuploidy within a biopsy. This study evaluates the sensitivity and specificity of segmental aneuploidy detection by three commercially available CCS platforms utilizing a cell line mixture model of segmental mosaicism in a six-cell trophectoderm biopsy.

Methods Two cell lines with known karyotypes were obtained and mixed together at specific ratios of six total cells $(0: 6,1: 5,2: 4,3: 3,4: 2,5: 1$, and 6:0). A female cell line containing a $16.2 \mathrm{Mb}$ deletion on chromosome 5 and a male cell line containing a $25.5 \mathrm{Mb}$ deletion on chromosome 4 were used to create mixtures at each level. Six replicates of each mixture were prepared, randomized, and blinded for analysis by one of the three CCS platforms (SNP-array, VeriSeq NGS, or NexCCS). Sensitivity and specificity of segmental aneuploidy at each level of mosaicism was determined and compared between each platform. Additionally, an alternative VeriSeq NGS analysis
\end{abstract}

Nathan R. Treff

ntreff@rmanj.com

1 Reproductive Medicine Associates of New Jersey, 140 Allen Rd, Basking Ridge, NJ 07920, USA

2 Foundation for Embryonic Competence, 140 Allen Rd, Suite 300, Basking Ridge, NJ 07920, USA method utilizing previously published criteria was evaluated.

Results Examination of the default settings of each platform revealed that the sensitivity was significantly different between NexCCS and SNP up to $50 \%$ mosaicism, custom VeriSeq, and SNP-array up to $66 \%$ mosaicism, and between NexCCS and custom VeriSeq up to $50 \%$ mosaicism. However, no statistical difference was observed in mixtures with $>50 \%$ mosaicism with any platform. No comparison was made between default VeriSeq, as it does not report segmental imbalances. Furthermore, while the use of previously published criteria for VeriSeq NGS significantly increased sensitivity at low levels of mosaicism, a significant decrease in specificity was observed ( $66 \%$ false positive prediction of segmental aneuploidy).

Conclusion These results demonstrate the potential of NGS-based detection methods to detect segmental mosaicism within a biopsy. However, these data also demonstrate that a balance between sensitivity and specificity should be more carefully considered. These results emphasize the importance of vigorous preclinical evaluation of new testing criteria prior to clinical implementation providing a point of departure for further algorithm development and improved detection of mosaicism within preimplantation embryos.

Keywords Segmental aneuploidy $\cdot$ Comprehensive chromosome screening $\cdot$ Next generation sequencing . Mosaicism $\cdot$ Preimplantation genetic screening

\section{Introduction}

Since the improvement in amplification strategies and the development of the ability to accurately screen for and diagnose 
aneuploidy in all 24 human chromosomes, contemporary comprehensive chromosomal screening (CCS) methods have become well developed and are now a common, routine part of infertility care. Given that approximately half of human preimplantation embryos are abnormal and the fact that aneuploidy rates increase drastically with advanced maternal age [1], these important advancements in PGS have allowed for improved outcomes in select patients undergoing in vitro fertilization (IVF) [2-5].

In particular, much attention has been given to the detection and screening of whole chromosome aneuploidy. A variety of methods including qPCR, array-CGH, and next generation sequencing (NGS) have been developed in order to accurately screen embryos for use in IVF. Namely, the development of high-throughput, massively parallel sequencing for use with CCS has been on the forefront of much research and is now common in many clinics. This advance in technology correlates to high accuracy screening of multiple samples while maintaining low costs.

With such advances in technology, it is now even possible to screen embryos for sub-chromosomal imbalances including inherited unbalanced translocations and segmental aneuploidies utilizing methods such as SNP-array and NGS [6-9] and the detection of small segments has been reported [10-13]. Recently, it has been reported that the frequency of clinically significant de novo segmental imbalances is higher than originally thought (2.5\%) [14]. Recent data also suggests that the majority of segmental errors arise during mitosis, which leads to mosaicism [15]. Furthermore, recent data published by Kort et al. reported findings of several reciprocal segmental aneuploidies in discarded embryos that had been biopsied multiple times [16], leading us to suspect that segmental aneuploidy, when present, may be commonly found in a mosaic state.
The ability to detect segmental aneuploidy within a mosaic embryo may be possible when performing testing on a trophectoderm biopsy which contains multiple cells from the blastocyst. Mosaicism within the biopsy itself may result in altered, non-integer copy numbers and could be detectable with new CCS methodologies. This study sought to model the various levels of segmental mosaicism that might be observed in a typical trophectoderm biopsy and compare three current technologies with ability to detect sub-chromosomal imbalances to detect segmental aneuploidy in a mosaic sample.

\section{Materials and methods}

In order to create positive controls for specific levels of mosaicism, two cell lines containing known segmental deletions, GM14131 (46,XX,del(5)(p15.1).ish del(5)(p15.33p15.1) (D5S23-).arr 5p15.33p15.1(68519-16362247) $\times 1)$ and GM22601 (46,XY,del(4)(p15.2).arr 4p16.3p15.2(5566525591051) $\times 1$ ), were purchased from Coriell Cell Repository (Camden, NJ). Each cell line was previously characterized for karyotypes by the supplier. The cells were then cultured and passaged once as recommended by the supplier. Individual cells were collected under a dissecting microscope and mixed together at specific ratios of six total cells $(0: 6,1: 5,2: 4,3: 3$, $4: 2,5: 1$, and 6:0). Twelve replicates of each mixture were made and then equally and randomly divided between three CCS platforms for aneuploidy screening (Fig. 1).

Three CCS screening methods were examined in this study: (i) VeriSeq PGS (Illumina Inc., Santa Clara, CA), a commercially available method involving whole-genome amplification (WGA) and next generation sequencing (NGS) on a MiSeq; (ii) targeted next generation sequencing (NexCCS)
Fig. 1 Mixture model experimental strategy illustration for preparation of samples involving a male del(4) cell line and a female del(5) cell line where inverse changes in levels of each aneuploidy are expected. Cells are mixed in a single tube in known ratios of six total cells $(0: 6,1: 5$, $2: 4,3: 3,4: 2,5: 1$, and $6: 0)$ to mimic various levels of mosaicism in a trophectoderm biopsy

\section{Del(5);Del(4) Experimental Strategy}

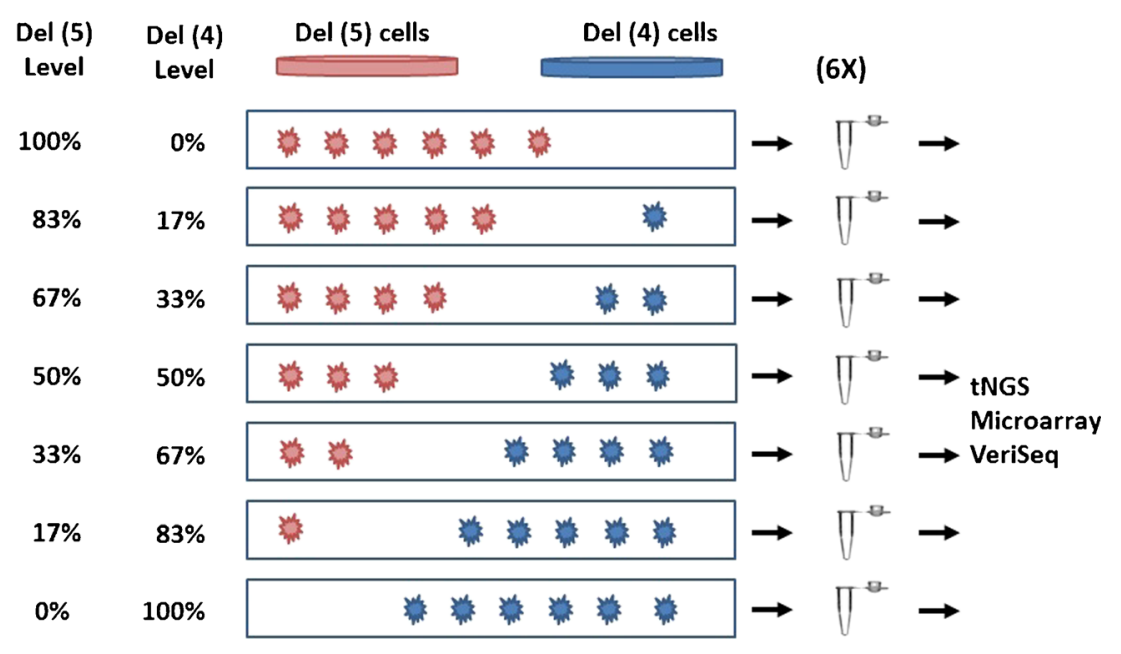



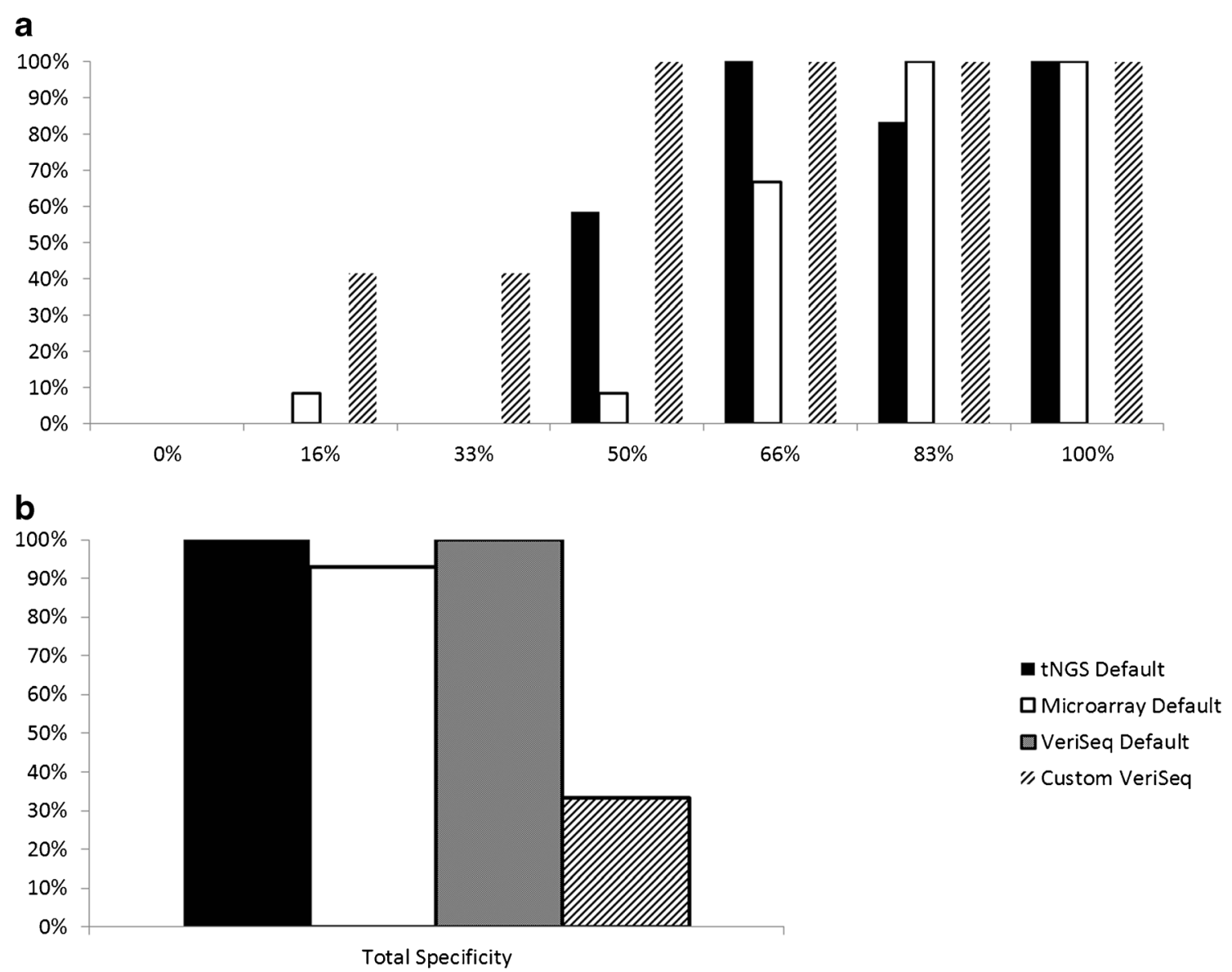

Fig. 2 a Sensitivity across three sets of analyses for each mixture level: custom NexCCS, custom SNP-array, VeriSeq default settings, and VeriSeq with criteria defined by Vera-Rodriguez et al. [13] (custom VeriSeq). Sensitivity is based on detecting segmental deletions at each

(Foundation for Embryonic Competence Inc.), another commercially available method involving targeted amplification and next generation sequencing on an Ion Torrent Proton sequencer; and (iii) SNP-array, a whole-genome amplificationbased method that uses arrays to assess aneuploidy [17]). Additionally, the first platform (VeriSeq NGS) was further evaluated utilizing previously published criteria by VeraRodriguez et al. [13]. Blinded computational segmental aneuploidy predictions were then made utilizing one of four level. b Specificity across all samples for the same four analysis methods based on the frequency of detecting a normal copy number for each of the remaining chromosomes known to be uniformly normal

criteria: (i) utilizing an in-house custom python algorithm to analyze the results from the Affymetix software (SNP-array); (ii) as recommended by the supplier utilizing the automatic aneuploidy calls made by Bluefuse Multi software (BlueFuse, Illumina Inc., version 4.2(20289)), termed "Default VeriSeq"; (iii) customized criteria for VeriSeq PGS (which examines every $10 \mathrm{Mb}$ of amplicons, observes changes in the median copy numbers, and overrides automated calls made by Bluefuse Multi software), termed "custom VeriSeq"and as

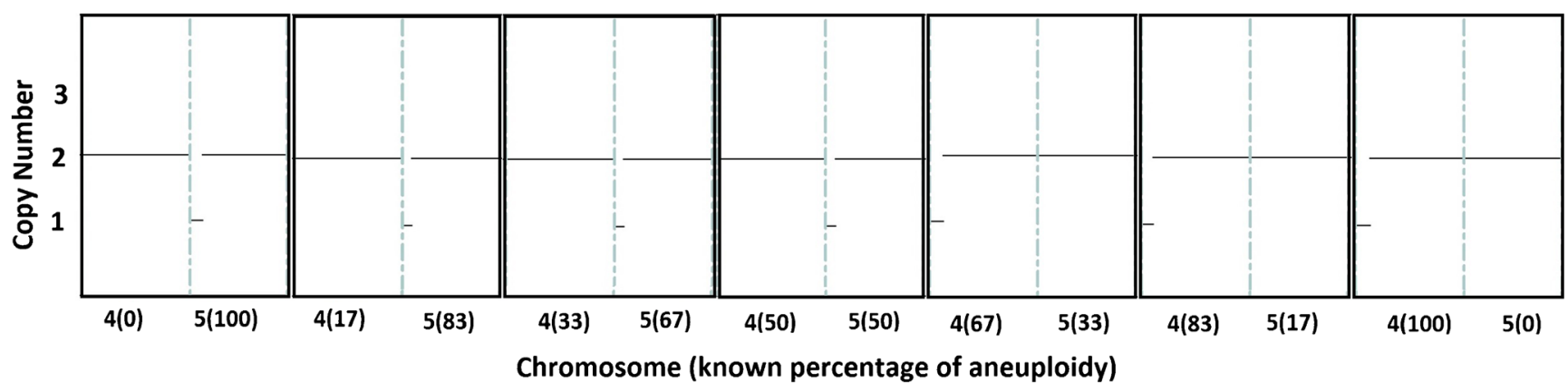

Fig. 3 Example plots from SNP-array analyses of the segmental del(4) and del(5) six-cell mixture sets. As the level of aneuploidy increases in the sample, there is a concomitant change in the copy number values of the chromosome segments of interest 


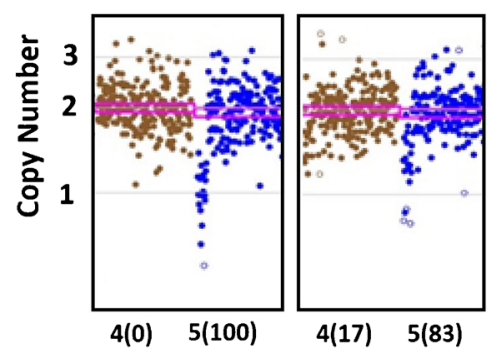

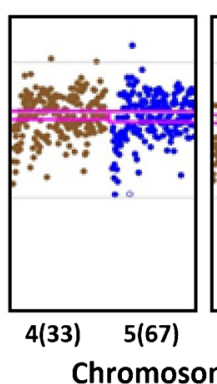

Chromosome

Fig. 4 Example plots from NexCCS analyses of the segmental del(4) and del(5) five six-cell mixture sets. As the level of aneuploidy increases in the sample, there is a concomitant change in the copy number values of

previously published [18]; and (iv) customized criteria for NexCCS, termed "NexCCS."

After the predictions of segmental aneuploidy were made, samples were then unblinded and evaluated for consistency with expected results. Sensitivity was defined as the percentage of samples which were predicted as abnormal for the correct chromosome segment depending on which mixture was tested (i.e., del(4) or del(5)) and was determined for each chromosome $(n=12)$ at each of the respective mixture levels for each platform and its analysis settings. Specificity was defined as the percentage of samples where euploidy was predicted for all the chromosomes and segments expected to be normal or disomic ( $n=42$ for each method: the no. of true positives for each mixture level multiplied by the no. of sets of samples (2)). The performance of each platform was then compared using a chi-squared test for statistical significance at each mixture level for its sensitivity and overall specificity.

\section{Results}

Analysis of the three CCS platforms demonstrated the ability to reliably predict an abnormality correctly at as low as $17 \%$ with

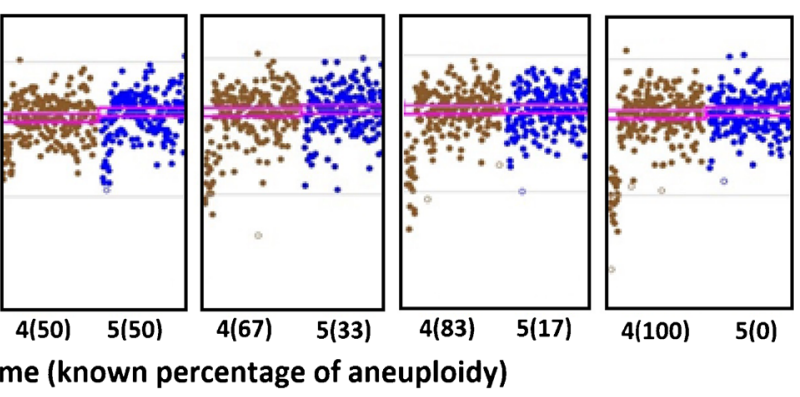

the chromosome segments of interest. This can be seen through the gradual migration of amplicons (represented by blue and orange dots) on chromosomes 4 and 5 in an inverse fashion

custom VeriSeq, $50 \%$ with custom NexCCS, and 50\% with SNP-array (Fig. 2). In all sample sets, increased detection of segmental errors was observed as the percentage of aneuploid cells increased in the mixture. Default VeriSeq settings, which utilize automated aneuploidy calls from Bluefuse Multi software do not report segmental aneuploidies and therefore did not detect segmental imbalances at any mixture level. Comparison of custom VeriSeq and NexCCS yielded significant differences at $17 \%(p=0.0119), 33 \%(p=0.0119)$, and $50 \%(p=0.0119)$; however, there was no significant difference at $66 \%(p=1), 83 \%(p=0.139)$, or $100 \%(p=1)$. Similarly, comparison between NexCCS and SNP-array yielded no significant statistical difference at $17 \%(p=0.307)$ or at $33 \%$ $(p=1)$. Significant statistical differences were observed at 50 and $66 \%(p=0.0093, p=0.0284)$; however, as aneuploidy increased in the sample $(83,100 \%)$, no significant differences were observed $(p=0.139, p=1)$. Lastly, comparison between SNP-array and custom VeriSeq resulted in statistical differences at 33\% ( $p=0.0119), 50 \%(P<0.0001)$, and 66\% $(p=0.0284)$, but no difference was observed at 88 or $100 \%(p=1)$ (Fig. 2). The overall specificity was $93 \%$ for custom SNP-array ( $p=0.0126$ ), and $100 \%$ for custom NexCCS and Default VeriSeq methods of analysis (Fig. 2).

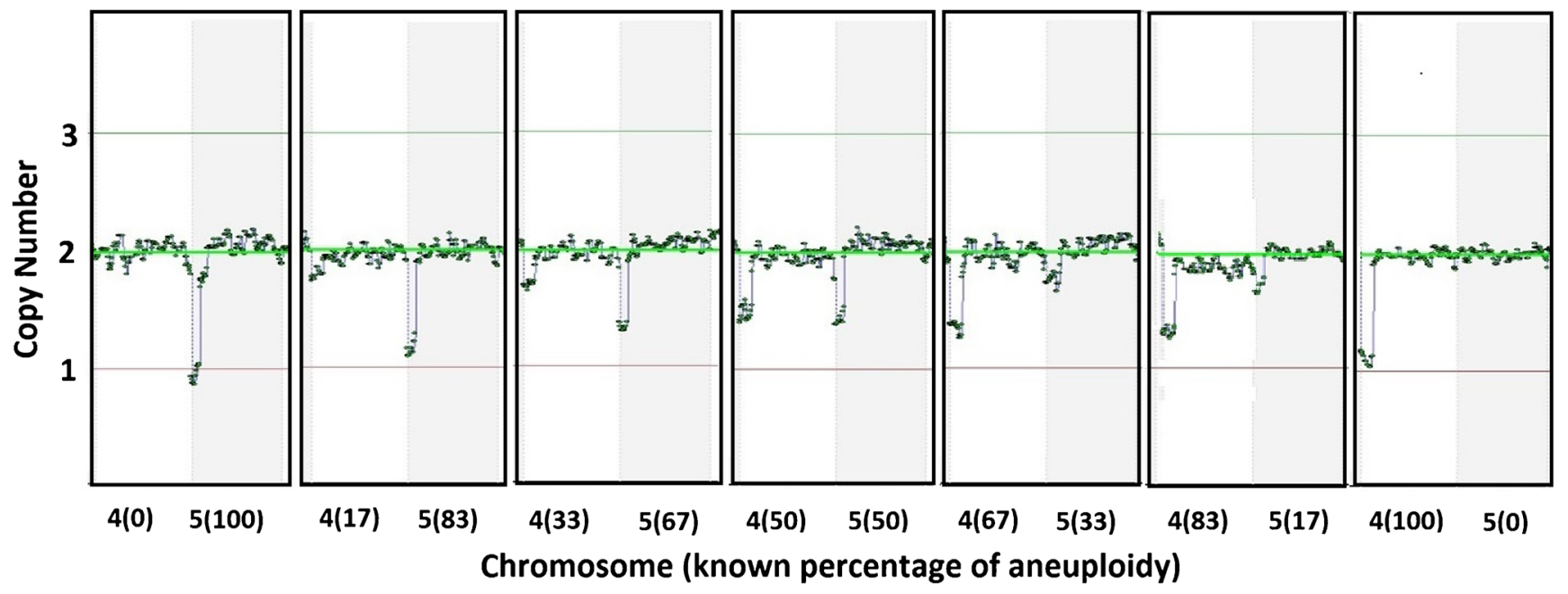

Fig. 5 Example plots from VeriSeq NGS analyses of the segmental del(4) and del(5) six-cell mixture sets. As the level of aneuploidy increases in the sample, there is a concomitant change in the copy number values of the chromosome segments of interest 
In contrast, when custom VeriSeq analysis criteria, as defined by Vera-Rodriguez et al. [13], were applied to the data set, significantly improved sensitivity of detecting aneuploidy was observed from 17 to $66 \%$ mosaicism levels $(p<0.05)$. Nevertheless, the subsequent improvement in sensitivity resulted in a greatly significant increase $(p<0.0001)$ in the rate of false positive segmental calls. The false positive rate increased from 7\% (6/84), using SNP-array, 0\% (0/84) with custom NexCCS and default VeriSeq analysis methods, to a stark 67\% (56/84) when utilizing the custom VeriSeq criteria (Fig. 2). It is important to note here that WGA-based amplification methods such as VeriSeq may provide better coverage of the genome; however, this comes at a significant cost to specificity. Furthermore, while target-based methods may provide less coverage of the genome and thus decreased sensitivity, they will display better overall specificity. This limitation can also make the diagnosis of de novo segmental imbalances in regions not covered by the sequencing panel difficult. Further refinement of the panel or inclusions of more targeted regions may help in addressing this problem.

These results clearly demonstrate the importance of balance between sensitivity and specificity when considering

Theoretical
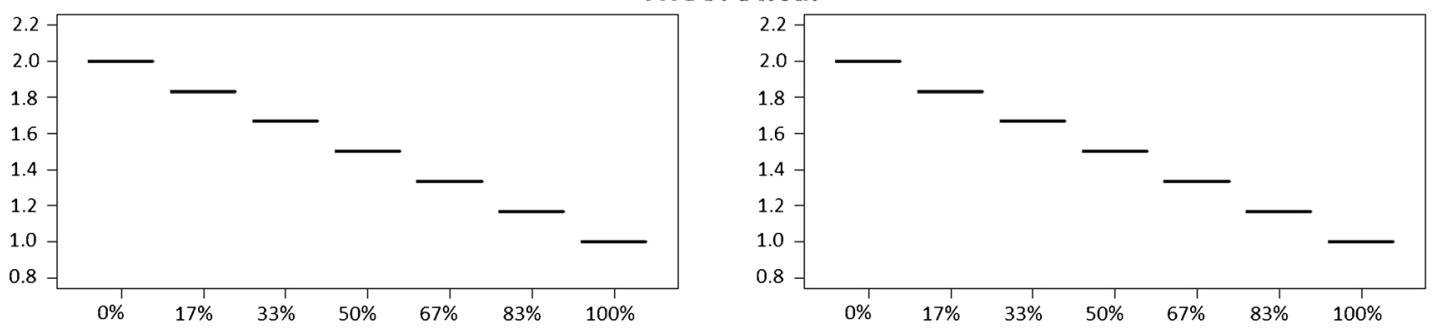

SNP Array
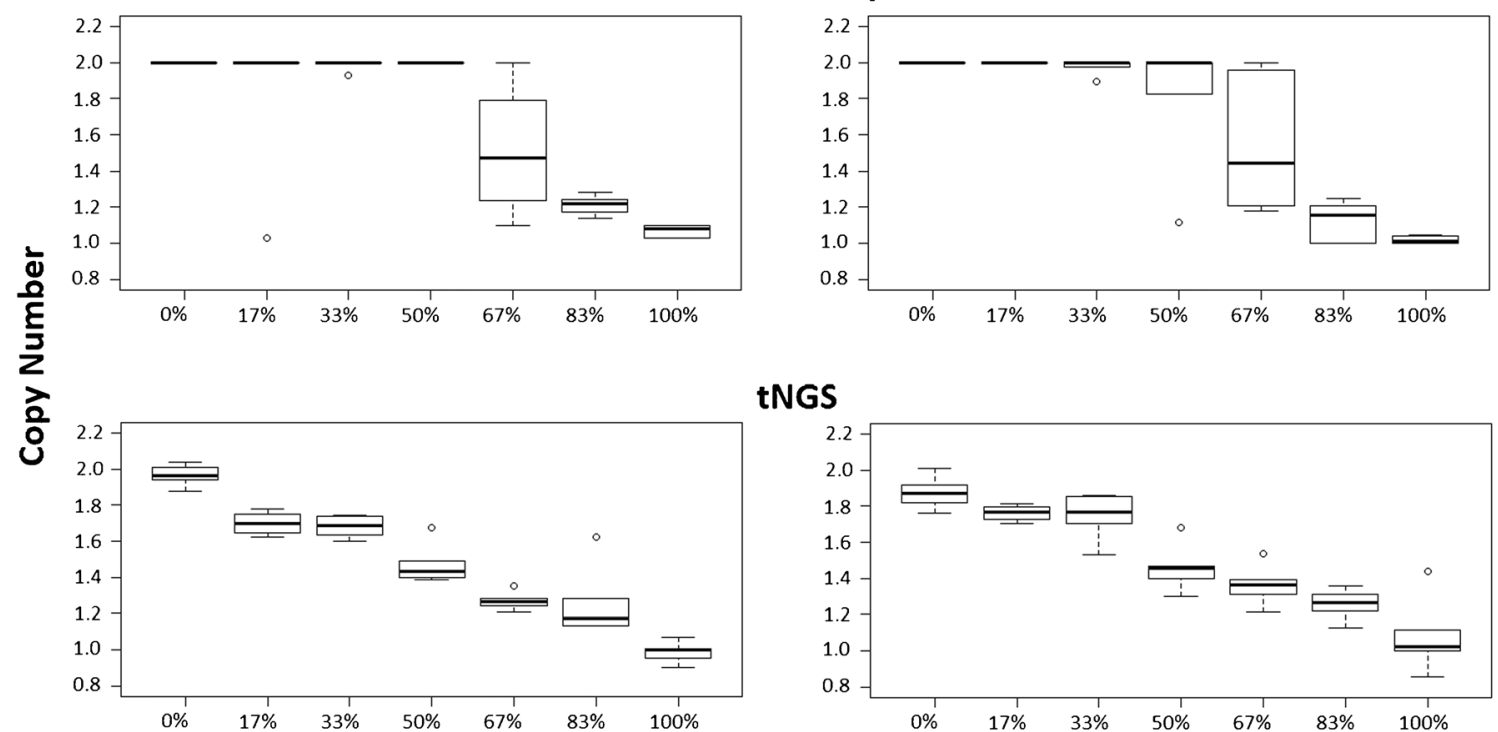

tNGS

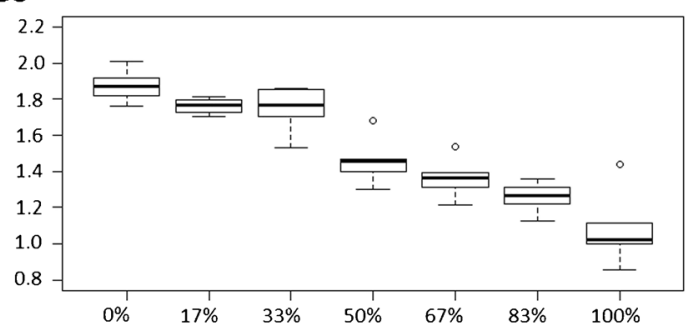

VeriSeq

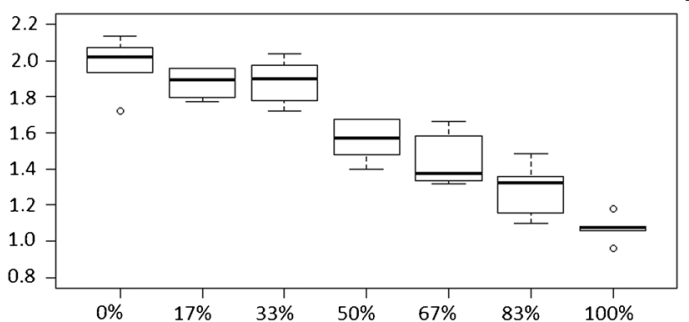

Percent chr 4 deletion

Fig. 6 Box and whisker plots showing the distribution and variance of copy number assignments for target mosaic chromosome segments as the percent of spike-in aneuploidy increases in the sample with each respective platform (SNP-array, NexCCS, and VeriSeq NGS). As the level of

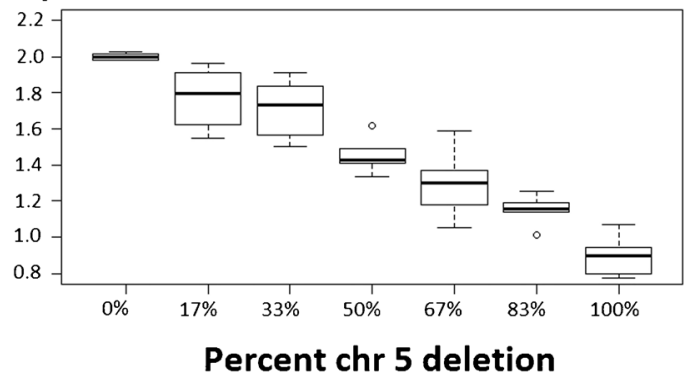

aneuploidy increases in the sample, there is an overall decrease in the copy number of the chromosome segments of interest (4 and 5). Included is a theoretical box plot illustrating the expected copy number change based on the contribution of chromosomes from each sample 
criteria for detection of segmental aneuploidy in a mosaic sample. Overall, two thirds of the samples gave similar false positives when applying previously published custom analysis criteria, illustrating further need to carefully evaluate criteria prior to its implementation in a clinical setting.

In order to illustrate the performance of each platform, example copy number plots for SNP-array, NexCCS, and VeriSeq are shown in Figs. 3, 4, and 5, which show the expected gradual change as the level of segmental aneuploidy increases in each mixture. SNP-array, which does not show intermediate copy numbers, only shows a change in the whole copy number assignments and migrates from disomy to monosomy. Reproducibility was considered by evaluating the distributions of copy number assignments for all replicates for both platforms and is shown in Fig. 6.

\section{Conclusions}

Recently, research into the ability of contemporary CCS platforms to detect mosaicism has been given considerable attention. Embryonic mosaicism (both whole chromosome and segmental) is a complicated problem, and there are still many important factors that must be considered prior to implementation of screening in the clinic $[19,20]$. Segmental aneuploi$\mathrm{dy}$ in particular represents a unique challenge in that the detection limits are much lower than those observed with whole chromosome mosaicism [21]. Additionally, other factors such as the size and location of the duplication/deletion may also affect the predictive value of the individual biopsy as well as the clinical outcome of the embryo. Furthermore, the distribution of mosaicism within the embryo may also impact the accuracy of such a test, as by definition a sampling error of the embryo will exist when only single biopsy is taken.

However, while these issues may be relevant, the purpose of this study was to focus on the limits of detection of the three platforms; namely, the percentage of cells within a multi-cell sample that need to be aneuploid to allow detection, how often a platform can detect the abnormal cells, and how often artifacts of the technology result in incorrectly predicted abnormalities. This study also only focuses on segmental aneuploidies that are $>5 \mathrm{Mb}$ as this detection limit has been previously validated with NGS [6]. This experimental design strategy was the foundation of two previous studies, the first of which examined the ability of NGS and qPCR to detect whole chromosome aneuploidy in a mosaic sample [21], and the second which sought to develop an accurate method of qPCR-based CCS for uniform aneuploidy [20]. Although criteria for detecting mosaicism was recently described [13] and represents an important first step into the investigation of mosaicism, further refinement of criteria is needed. This is clearly illustrated by the fact that although a significant increase in the sensitivity of detection was seen, it resulted in a drastic loss $(66 \%)$ of specificity. In that, in two thirds of the samples analyzed, a false positive was reported.

While defining the specificity and sensitivity of a method is important to the development of an accurate model of mosaicism, additional considerations must be given prior to implementation in the clinic as a diagnostic tool [22]. These include the distribution of mosaicism in the remaining embryo, the size of the segmental imbalance, the identity of the chromosome affected, and the actual clinical outcomes. Further preclinical testing should include evaluating multiple biopsies of the same embryo in order to establish the predictive value of a single biopsy for the remaining embryo [23]. Next, a prospective, blinded, non-selection study should be performed to establish positive and negative predictive values of a diagnosis for actual clinical outcomes [24]. Finally, new clinical interventions should work towards randomized clinical trials ultimately to establish the efficacy of a diagnosis of mosaicism as a predictor of reproductive outcome [15].

Open Access This article is distributed under the terms of the Creative Commons Attribution 4.0 International License (http:// creativecommons.org/licenses/by/4.0/), which permits unrestricted use, distribution, and reproduction in any medium, provided you give appropriate credit to the original author(s) and the source, provide a link to the Creative Commons license, and indicate if changes were made.

\section{References}

1. Franasiak JM, et al. The nature of aneuploidy with increasing age of the female partner: a review of 15,169 consecutive trophectoderm biopsies evaluated with comprehensive chromosomal screening. Fertil Steril. 2014;101(3):656-63. e1

2. Forman EJ, et al. Single embryo transfer with comprehensive chromosome screening results in improved ongoing pregnancy rates and decreased miscarriage rates. Hum Reprod. 2012;27(4):1217-22.

3. Scott Jr RT, et al. Blastocyst biopsy with comprehensive chromosome screening and fresh embryo transfer significantly increases in vitro fertilization implantation and delivery rates: a randomized controlled trial. Fertil Steril. 2013;100(3):697-703.

4. Yang Z, et al. Selection of single blastocysts for fresh transfer via standard morphology assessment alone and with array CGH for good prognosis IVF patients: results from a randomized pilot study. Mol Cytogenet. 2012;5(1):24.

5. Forman EJ, et al. Comprehensive chromosome screening alters traditional morphology-based embryo selection: a prospective study of 100 consecutive cycles of planned fresh euploid blastocyst transfer. Fertil Steril. 2013;100(3):718-24.

6. Bono S, et al. Validation of a semiconductor next-generation sequencing-based protocol for preimplantation genetic diagnosis of reciprocal translocations. Prenat Diagn. 2015;35(10):938-44.

7. Ou J, et al. Identification of small segmental translocations in patients with repeated implantation failure and recurrent miscarriage using next generation sequencing after in vitro fertilization/ intracytoplasmic sperm injection. Mol Cytogenet. 2015;8(1):105.

8. Zhang W, et al. Clinical application of next-generation sequencing in preimplantation genetic diagnosis cycles for Robertsonian and reciprocal translocations. J Assist Reprod Genet. 2016;33(7):899_ 906. 
9. Xiong B, et al. Using SNP array to identify aneuploidy and segmental imbalance in translocation carriers. Genom Data. 2014;2: 92-5.

10. Treff NR, et al. Use of single nucleotide polymorphism microarrays to distinguish between balanced and normal chromosomes in embryos from a translocation carrier. Fertil Steril. 96(1):e58-65.

11. Treff NR, et al. SNP array-based analyses of unbalanced embryos as a reference to distinguish between balanced translocation carrier and normal blastocysts. J Assist Reprod Genet. 2016;33(8):1115-9.

12. Wang L, et al. Validation of copy number variation sequencing for detecting chromosome imbalances in human preimplantation embryos. Biol Reprod. 2014;91(2):37.

13. Vera-Rodriguez M, et al. Distribution patterns of segmental aneuploidies in human blastocysts identified by next-generation sequencing. Fertil Steril. 2016;105(4):1047-55.

14. Wapner RJ, et al. Chromosomal microarray versus karyotyping for prenatal diagnosis. N Engl J Med. 2012;367(23):2175-84.

15. Werner MD, et al. Targeted NGS provides accurate predictions of segmental (SEG) aneuploidy and prognosticates reduced reproductive potential of the human blastocyst. Fertil Steril. 2016;106(3): e68.

16. Kort DH, et al. Human embryos commonly form abnormal nuclei during development: a mechanism of DNA damage, embryonic aneuploidy, and developmental arrest. Hum Reprod. 2016;31(2): 312-23.
17. Treff NR, et al. Accurate single cell 24 chromosome aneuploidy screening using whole genome amplification and single nucleotide polymorphism microarrays. Fertil Steril. 2010;94(6):2017-21.

18. Vera-Rodriguez M., et al. Distribution patterns of segmental aneuploidies in human blastocysts identified by next-generation sequencing. Fertil Steril. 2016.

19. Taylor TH, et al. The origin, mechanisms, incidence and clinical consequences of chromosomal mosaicism in humans. Hum Reprod Update. 2014;20(4):571-81.

20. Treff NR, et al. Development and validation of an accurate quantitative real-time polymerase chain reaction-based assay for human blastocyst comprehensive chromosomal aneuploidy screening. Fertil Steril. 2012;97(4):819-24.

21. Goodrich D, et al. A randomized and blinded comparison of qPCR and NGS-based detection of aneuploidy in a cell line mixture model of blastocyst biopsy mosaicism. J Assist Reprod Genet. 2016.

22. Scott Jr RT, Galliano D. The challenge of embryonic mosaicism in preimplantation genetic screening. Fertil Steril. 2016;105(5):1150-2.

23. Juneau CR, et al. Reliable detection of segmental aneuploidy identified by next generation sequencing (NGS). Fertil Steril. 2016;106(3):e377.

24. Scott Jr RT, et al. Comprehensive chromosome screening is highly predictive of the reproductive potential of human embryos: a prospective, blinded, nonselection study. Fertil Steril. 2012;97(4):870-5. 